\title{
The Goryachev-Chaplygin Top and the Toda Lattice
}

\author{
C. Bechlivanidis ${ }^{1}$ and P. van Moerbeke ${ }^{1,2, \star}$ \\ ${ }^{1}$ Department of Mathematics, University of Louvain, B-1348 Louvain-la-Neuve, Belgium \\ ${ }^{2}$ Department of Mathematics, Brandeis University, Waltham, MA 02254, USA
}

\begin{abstract}
The Goryachev-Chaplygin top is a rigid body rotating about a fixed point with principal moments of inertia $A, B, C$ satisfying $A=B=4 C$ and with center of mass lying in the equatorial plane. The problem is algebraically completely integrable as a linear flow on a hyperelliptic Jacobian, only upon putting the principal angular momentum in the horizontal plane.

This system admits asymptotic solutions with fractional powers in $t$ and depending on 4 degrees of freedom. As a consequence, the affine invariant surfaces of the Chaplygin top are double covers of the hyperelliptic Jacobian above, ramified along two translates of the theta divisor, touching in one point. This system is an instance of a (master) system of differential equations in 7 unknowns having 5 quadratic constants of motion; a careful analysis of this system reveals an intimate (rational) relationship with the 3-body periodic Toda lattice.
\end{abstract}

The Goryachev-Chaplygin top is a rigid body rotating about a fixed point with principal moments of inertia $A, B, C$ satisfying $A=B=4 C$ and with center of mass lying in the equatorial plane (through the fixed point) going with the moments $A$ and $B$. First introduced by Goryachev [7] in 1900 and later integrated by Chaplygin [5] in terms of hyperelliptic integrals, we learned about the system from Golubev [6]. This motion has an extra-constant of motion, only upon putting the principal angular momentum in the horizontal plane. After some rescaling, the equations of motion take on the form:

$$
\begin{array}{ll}
\dot{x}=3 y z, & \dot{u}=4 z v-y w, \\
\dot{y}=-3 x z-4 w, & \dot{v}=x w-4 z u, \\
\dot{z}=4 v, & \dot{w}=y u-x v .
\end{array}
$$

\footnotetext{
* The support of National Science Foundation grant No. DMS-8403136 is gratefully acknowledged. I thank Victor Guillemin for mentioning this problem
} 
with energy

$$
x^{2}+y^{2}+4 z^{2}-8 u=6 a,
$$

and trivial invariants

$$
\begin{gathered}
u^{2}+v^{2}+w^{2}=c, \\
x u+y v+z w=\text { const } .
\end{gathered}
$$

Only upon putting this constant equal to 0 , does the system have an extrainvariant

$$
\left(x^{2}+y^{2}\right) z+4 x w=2 b .
$$

The system (1) is weight-homogeneous with $x, y, z$ having weight 1 and $u, v, w$ having weight 2 . Then being algebraically completely integrable on twodimensional complex tori $T_{\mathbb{C}}^{2}$, such a system is commonly believed to have Laurent solutions with simple poles for $x, y, z$ and double poles for $u, v, w$, depending on 4 degrees of freedom, namely the values $a, b, c$ of the constant of motion and the local parameter of the algebraic curve(s) on $T_{\mathbb{C}}^{2}$ along which the variables blow up. This system is interesting in that it is different: for the system to admit asymptotic solutions depending on 4 degrees of freedom, the leading powers must be as follows

$$
(x, y, z, u, v, w)=\left(t^{-3 / 2}, t^{-3 / 2}, t^{-1}, t^{-2}, t^{-2}, t^{-1 / 2}\right) \times \text { a Taylor series } .
$$

This phenomenon will be explained in this paper by showing that the GoryachevChaplygin equations are part of a system of differential equations in seven unknowns having five quadratic constants of motion. This extended system also shows that the Goryachev-Chaplygin top is intimately related to the three-body Toda lattice.

Consider indeed the following system of 7 differential equations in the unknowns $y_{1}, \ldots, y_{7}$ :

$$
\begin{array}{cc}
X_{1}: \dot{y}_{1}=-8 y_{7}, & \dot{y}_{4}=4 y_{2} y_{5}-y_{7}, \\
\dot{y}_{2}=4 y_{5}, & \dot{y}_{5}=y_{6}-4 y_{2} y_{4}, \\
\dot{y}_{3}=2\left(y_{4} y_{7}-y_{5} y_{6}\right), & \dot{y}_{6}=-y_{1} y_{5}+2 y_{2} y_{7}, \\
\dot{y}_{7}=y_{1} y_{4}-2 y_{2} y_{6}-4 y_{3} .
\end{array}
$$

The following five quadrics are constants of motion for this system:

$$
\begin{aligned}
& Q_{1}=y_{1}+4 y_{2}^{2}-8 y_{4}=6 a, \\
& Q_{2}=y_{1} y_{2}+4 y_{6}=2 b, \\
& Q_{3}=y_{4}^{2}+y_{5}^{2}+y_{3}=c, \\
& Q_{4}=y_{4} y_{6}+y_{5} y_{7}+y_{2} y_{3}=d, \\
& Q_{5}=y_{6}^{2}+y_{7}^{2}-y_{1} y_{3}=e .
\end{aligned}
$$


Another flow commuting with (3) has the form

$$
\begin{array}{cl}
X_{2}: \dot{y}_{1}=4\left(y_{1} y_{5}+2 y_{2} y_{7}\right), & \dot{y}_{4}=\frac{1}{2} y_{1} y_{5}-y_{2} y_{7}, \\
\dot{y}_{2}=-2 y_{7}, & \dot{y}_{5}=-\frac{1}{2} y_{1} y_{4}+y_{2} y_{6}+2 y_{3}, \\
\dot{y}_{3}=2 y_{2}\left(y_{4} y_{7}-y_{5} y_{6}\right)-4 y_{3} y_{5}, & \dot{y}_{6}=-y_{2}\left(y_{1} y_{5}+2 y_{2} y_{7}\right)+\frac{1}{2} y_{1} y_{7}, \\
\dot{y}_{7}=y_{2}\left(y_{1} y_{4}+2 y_{2} y_{6}\right)-\frac{1}{2} y_{1} y_{6}+4 y_{2} y_{3} .
\end{array}
$$

Although the system (3) is not weight-homogeneous, it admits Laurent solutions depending on 6 free parameters $a, b, c, d, e$, and $Z$, to wit:

$$
\begin{aligned}
& y_{1}=-\frac{2 \varepsilon Z}{t}+2 Z^{2}-2 \varepsilon\left(Z\left(Z^{2}-2 a\right)+W\right) t-(2 P+W) Z t^{2}+O\left(t^{3}\right), \\
& y_{2}=-\frac{\varepsilon}{2 t}-\frac{Z}{2}-\frac{\varepsilon}{2}\left(Z^{2}-2 a\right) t-\frac{t^{2}}{4}(2 P+3 W)+O\left(t^{3}\right), \\
& y_{3}=\frac{\varepsilon}{8 t}(P+W)+\frac{3 Z}{8}(P+W)-\frac{\varepsilon t}{8}\left(\left(5 Z^{2}-a\right)(P+W)-8(2 c Z+d)\right)+O\left(t^{2}\right), \\
& y_{4}=-\frac{1}{8 t^{2}}+\frac{Z^{2}-2 a}{8}+\frac{\varepsilon t}{8}(2 P+W)+O\left(t^{2}\right), \\
& y_{5}=\frac{\varepsilon}{8 t^{2}}-\frac{\varepsilon\left(Z^{2}-2 a\right)}{8}-\frac{t}{8}(2 P+3 W)+O\left(t^{2}\right), \\
& y_{6}=\frac{Z}{4 t^{2}}+\frac{1}{4}\left(2 P-\left(Z^{2}-2 a\right) Z+W\right)-\frac{t \varepsilon Z}{4}(2 P+3 W)+O\left(t^{2}\right), \\
& y_{7}=-\frac{\varepsilon Z}{4 t^{2}}+\frac{\varepsilon}{4}\left(Z\left(Z^{2}-2 a\right)+W\right)+\frac{t Z}{4}(2 P+W)+O\left(t^{2}\right),
\end{aligned}
$$

where $\varepsilon^{2}=-1$ and

$$
P(Z) \equiv 2 Z^{3}-3 a Z+b, \quad Q(Z) \equiv 4 c Z^{2}+4 d Z+e,
$$

and where the parameters $(Z, W)$ belong to a hyperelliptic curve

$$
\mathscr{H}: W^{2}=P^{2}-4 Q .
$$

The Laurent solutions are thus parametrized by two copies $\mathscr{H}_{+}$and $\mathscr{H}_{-}$of the same hyperelliptic curve $\mathscr{H}$ for $\varepsilon= \pm i$. In order to embed $\mathscr{H}$ into some projective space, we search for functions $y_{0}, y_{1}, \ldots, y_{N}$ of increasing degree in the original variables having the property that the embedding $D$ of $\mathscr{H}_{+}+\mathscr{H}_{-}$into $\mathbb{P}^{N}$ via those functions satisfies the relation (see Adler and van Moerbeke $[1,4]$ ),

$$
\text { geometric genus }(2 D)=N+2 \text {. }
$$

This occurs for the first time for $N=15$; indeed the original set of variables $y_{0}, \ldots, y_{7}$ can be enlarged by adjoining 8 other functions, with the following 
Laurent series obtained from (5):

$$
\begin{aligned}
& y_{8}=y_{2} y_{3}=\frac{1}{16 t^{2}}(P+W)-\frac{\varepsilon Z}{4 t}(P+W)+O(1), \\
& y_{9}=y_{1} y_{3}=\frac{Z}{4 t^{2}}(P+W)-\frac{\varepsilon Z^{2}}{2 t}(P+W)+O(1), \\
& y_{10}=y_{1} y_{4}+2 y_{2} y_{6}=-\frac{Z^{2}}{2 t^{2}}-\frac{\varepsilon P}{2 t}+O(1), \\
& y_{11}=y_{1} y_{5}+2 y_{2} y_{7}=\frac{\varepsilon Z^{2}}{2 t^{2}}+\frac{W}{2 t}+O(1), \\
& y_{12}=-y_{5} y_{6}+y_{4} y_{7}=-\frac{\varepsilon(P+W)}{16 t^{2}}+O(1), \\
& y_{13}=y_{2} y_{12}-2 y_{3} y_{5}=-\frac{\varepsilon Z(P+W)}{16 t^{2}}+O\left(\frac{1}{t}\right), \\
& y_{14}=y_{3}^{2}=-\left(\frac{P+W}{8 t}\right)^{2}+\frac{3 \varepsilon Z(P+W)^{2}}{32 t}+O(1), \\
& y_{15}=y_{1} y_{12}+4 y_{3} y_{7}=-\frac{\varepsilon Z^{2}(P+W)}{2 t^{2}}+O\left(\frac{1}{t}\right) .
\end{aligned}
$$

Using these functions $y_{0}, \ldots, y_{15}$, one embeds each of the curves $\mathscr{H}_{+}$and $\mathscr{H}_{-}$into $\mathbb{P}^{15}$; thus embedded, they have only one point in common, at which they are

\section{Fig. 1}

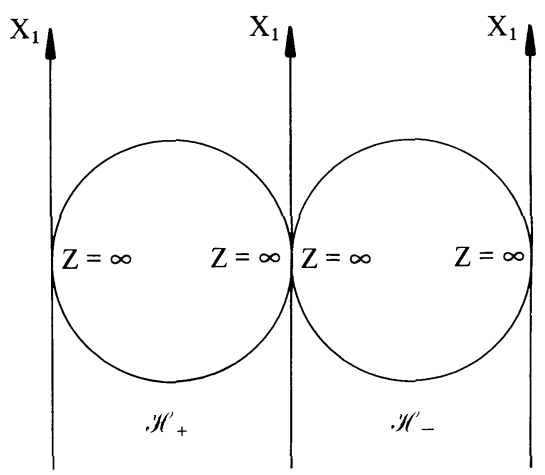

tangent to each other as illustrated in Fig. 1. Each of the curves have 2 points covering $Z=\infty$, at which $P+W$ behaves as follows:

$$
\begin{aligned}
P+W & =4 Z^{3}+O(Z), \text { by picking the }+ \text { sign for } W, \\
& =\frac{Q(Z)}{Z^{3}}+\text { lower order terms, by picking the }- \text { sign for } W .
\end{aligned}
$$

Then by picking the + sign for $W$ and by dividing the vector $\left(y_{0}, \ldots, y_{15}\right)$ with $y_{14}=y_{3}^{2}$, the corresponding point is mapped into the point $(0, \ldots, 0,1,0)$ in $\mathbb{P}^{15}$, 
which is independent of $\varepsilon$ and thus is common to $\mathscr{H}_{+}$and $\mathscr{H}_{-}$, whereas picking the - sign leads to two different points, according to the sign of $\varepsilon$. The divisor $D$ obtained in this way has genus 5 and thus $2 D$ has genus 17, satisfying the requirement (7). Following the methods in Adler and van Moerbeke [1,4], one attaches the affine part of the intersection of the five quadrics so as to obtain a smooth compact surface in $\mathbb{P}^{15}$ equipped with two commuting vector fields. One copy of $\mathscr{H}$ defines on $T^{2}$ a polarization $\left(\delta_{1}, \delta_{2}\right)$ with $\delta_{1} \delta_{2}=\operatorname{genus}(\mathscr{H})-1=1$, implying $\delta_{1}=\delta_{2}=1$; thus $T^{2}$ is principally polarized and it is the Jacobian of the hyperelliptic curve $\mathscr{H}$.

Let $d t_{1}$ and $d t_{2}$ be the two holomorphic 1 -forms on $T^{2}$ corresponding to the vector fields (3) and (4), i.e., $d t_{i}\left(X_{j}\right)=\delta_{i j}$. Then differentiating $y_{1} / y_{2}$ and $1 / y_{2}$ with regard to $t_{1}$ and $t_{2}$ and using the vector fields, one computes the restrictions $\omega_{1}$ and $\omega_{2}$ of $d t_{1}$ and $d t_{2}$ to $D=\mathscr{H}_{+}+\mathscr{H}_{-}$; as expected they lead to the hyperelliptic differentials

$$
\omega_{1}=\frac{Z d Z}{W} \quad \text { and } \quad \omega_{2}=\frac{d Z}{W},
$$

up to some multiplicative constants. The zeroes of $\omega_{2}$ provide the points where the vector field $X_{1}$ is tangent to the curves $\mathscr{H}_{+}$and $\mathscr{H}_{-}$on $T^{2}$. In particular $X_{1}$ is tangent to $\mathscr{H}_{+}$and $\mathscr{H}_{-}$at the point where both curves touch, as illustrated in Fig. 1.

The hyperelliptic curve $\mathscr{H}$ also plays another role: consider the projective linear span

$$
V=\left\{\sum \lambda_{i} \bar{Q}_{i}, \lambda_{i} \in \mathbb{C}\right\} \simeq \mathbb{P}^{4}
$$

of the 5 quadrics $\bar{Q}_{i}$, obtained by making the $Q_{i}$ homogeneous; for instance,

$$
\bar{Q}_{1}=y_{1} y_{0}+4 y_{2}^{2}-8 y_{4} y_{0}-6 a y_{0}^{2} \text {. }
$$

Then, we have the following identity

$$
\begin{aligned}
U \bar{Q}_{1} & -\frac{U}{Z} \bar{Q}_{2}+4 Z \bar{Q}_{3}+4 \bar{Q}_{4}+\frac{1}{Z} \bar{Q}_{5} \\
= & \left(2 \sqrt{Z} y_{4}+\frac{1}{\sqrt{Z}} y_{6}-\frac{2 U}{\sqrt{Z}} y_{0}\right)^{2}+\left(2 \sqrt{Z} y_{5}+\frac{1}{\sqrt{Z}} y_{7}\right)^{2} \\
& +\left(2 \sqrt{U} y_{2}-\frac{\sqrt{U}}{4 Z} y_{1}+\frac{y_{3}}{\sqrt{U}}\right)^{2}-\left(2 Z \sqrt{U} y_{0}-\frac{\sqrt{U}}{4 Z} y_{1}-\frac{1}{\sqrt{U}} y_{3}\right)^{2}
\end{aligned}
$$

in $y_{i} y_{j}$, except for the coefficient of $y_{0}^{2}$; identifying this coefficient leads to the equation

$$
4 U^{2}-2 P U+Q=0 .
$$

Its solution is given by $4 U=P+W$, and therefore the hyperelliptic curve $\mathscr{H}$ defined in (6) has a concrete representation as the curve of rank 4 quadrics in the span $V$ of the 5 quadrics $Q_{i}$. It shows that the curve of rank 4 quadrics ties up very closely with the set of curves which completes the affine intersection $\bigcap_{1}^{5}\left\{\bar{Q}_{i}=0, y_{0} \neq 0\right\}$ into an Abelian surface; for an explanation of this phenomenon, see [2]. 
1. Consider now the case $d=e=0$ and consider the transformation $\left(y_{2}, y_{3}, y_{4}, y_{5}, y_{6}, y_{7}\right) \curvearrowright(x, y, z, u, v, w)$ defined by

$$
W=\sqrt{y_{3}}, X=y_{6} / W, Y=y_{7} / W, Z=y_{2}, U=y_{4}, V=y_{5} .
$$

Since the function $y_{3}$ has a simple pole along the divisor $D=\mathscr{H}_{+}+\mathscr{H}_{-}$, and a double zero along a hyperelliptic curve of genus 2 , the transformation (9) defines a double cover of $T^{2}$ ramified along $D$. Using the last invariant, we obtain $y_{1}=x^{2}+y^{2}$, and the inverse transform reads

$$
\left(y_{1}, \ldots, y_{7}\right)=\left(x^{2}+y^{2}, z, w^{2}, u, v, x w, y w\right) .
$$

Putting this inverse transform into the constants of motion $Q_{1}, \ldots, Q_{4}$ leads to the 4 relations:

$$
\begin{gathered}
x^{2}+y^{2}+4 z^{2}-8 u=6 a, \\
\left(x^{2}+y^{2}\right) z+4 x w=2 b, \\
u^{2}+v^{2}+w^{2}=c, \\
w(x u+y v+z w)=0,
\end{gathered}
$$

whereas the last invariant $Q_{5}$ leads to an identity. Away from the locus $w=0$, we have

$$
x u+y v+z w=0 .
$$

Using the differential equations (3) for $y_{2}, y_{3}, y_{4}$, and $y_{5}$ and those for $y_{6}=x w$ and $y_{7}=y w$ combined with (11) leads to the following system of differential equations

$$
\begin{array}{ll}
\dot{x}=3 y z, & \dot{u}=4 z v-y w, \\
\dot{y}=3 x z-4 w, & \dot{v}=x w-4 z u, \\
\dot{z}=4 v, & \dot{w}=y u-x v .
\end{array}
$$

These are exactly Eqs. (1) for the Goryachev-Chaplygin top. The invariant surfaces defined by Eqs. (10) are double covers of the Jacobian of $\mathscr{H}$, ramified along the two hyperelliptic curves $\mathscr{H}_{+}$and $\mathscr{H}_{-}$. This explains why the asymptotic solutions to the differential equations (1) contain fractional powers. The asymptotic solutions (2) can be read off from (5) and the change of variables (9).

2. The next case under consideration is $c=d=0$, which we show is equivalent to the 3-body periodic Toda lattice. The latter motion is derived from the Hamiltonian

$$
H=\frac{1}{2} \sum_{1}^{3} p_{i}^{2}+\sum_{1}^{3} e^{q_{i}-q_{i+1}}
$$

which using Flaschka's transformation $z_{i+3}=-p_{i}$ and $z_{i}=\exp \left(q_{i}-q_{i+1}\right)$, is governed by the equations

$$
\begin{array}{ll}
\dot{z}_{1}=z_{1}\left(z_{4}-z_{5}\right), & \dot{z}_{4}=z_{1}-z_{3}, \\
\dot{z}_{2}=z_{2}\left(z_{5}-z_{6}\right), & \dot{z}_{5}=z_{2}-z_{1}, \\
\dot{z}_{3}=z_{3}\left(z_{6}-z_{4}\right), & \dot{z}_{6}=z_{3}-z_{2},
\end{array}
$$


with constants of motion

$$
\begin{aligned}
& Q_{1}^{\prime}=z_{1} z_{2} z_{3}=A, \\
& Q_{2}^{\prime}=z_{4}+z_{5}+z_{6}=B=0, \\
& Q_{3}^{\prime}=\frac{1}{2}\left(z_{4}^{2}+z_{5}^{2}+z_{6}^{2}\right)-z_{1}-z_{2}-z_{3}=C, \\
& Q_{4}^{\prime}=z_{4} z_{5} z_{6}+z_{1} z_{6}+z_{2} z_{4}+z_{3} z_{5}=D .
\end{aligned}
$$

Observe we may put the total momentum equal to zero $(B=0)$, without loss of generality. It is also well known that this problem linearizes on the Jacobi variety of a hyperelliptic curve [8] and it was shown by Adler and van Moerbeke [3] that the affine variety defined by the 4 constants completes into this Jacobian by adjoining 3 copies of the same hyperelliptic curve, each pair having one point in common (tacnode), as illustrated by Fig. 2.

Fig. 2

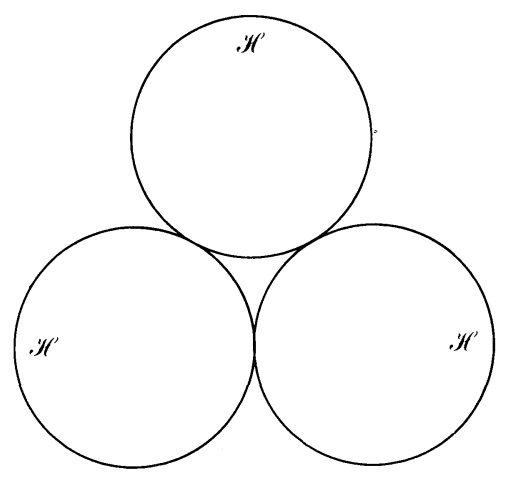

The embedding of the tori which complete the affine surface, defined by (12), can be done by means of the functions

$$
\left(1, z_{5}, z_{6}, z_{1}+z_{4} z_{5}, z_{2}+z_{5} z_{6}, z_{2} z_{4}-z_{5}\left(z_{1}+z_{4} z_{5}\right), z_{1} z_{2}, z_{2} z_{3}, z_{3} z_{1}\right) \text {. }
$$

They span the space of functions which blow up like $\frac{1}{t}$ at worst. Now pick the linear combinations which have a simple pole along two specific hyperelliptic curves and which remain finite along the third one; the only three such functions are $z_{1}+z_{4} z_{5}, z_{6}$, and $z_{2} z_{3}$. Then comparing their Laurent series with the ones of $y_{1}$, $y_{2}$, and $y_{3}$ leads to the following change of variables

$$
\begin{array}{cl}
y_{1}=z_{1}+z_{4} z_{5}, & y_{2}=-\frac{i z_{6}}{2}, \\
y_{3}=-\frac{z_{2} z_{3}}{16}, \quad y_{4}=-\frac{1}{8}\left(z_{2}+z_{3}\right), \\
y_{5}=\frac{i}{8}\left(z_{2}-z_{3}\right), \quad y_{6}=-\frac{i}{8}\left(z_{3} z_{5}+z_{2} z_{4}\right), \\
y_{7}=\frac{1}{8}\left(z_{3} z_{5}-z_{2} z_{4}\right) .
\end{array}
$$


In turn the 6 variables $z_{1}, \ldots, z_{6}$ can also be expressed rationally in terms of $y_{1}, \ldots, y_{7}$. Using the transformation (13) in the constants of motion $Q_{i}$ of the system (3), leads to the identities

$$
\begin{gathered}
Q_{1}=-Q_{3}^{\prime}+\frac{1}{2}\left(z_{4}+z_{5}-z_{6}\right) Q_{2}^{\prime}, \quad Q_{2}=-\frac{i}{2} Q_{4}^{\prime} \\
Q_{3}=0, \quad Q_{4}=\frac{i}{32} z_{2} z_{3} Q_{2}^{\prime}, \quad Q_{5}=\frac{1}{16} Q_{1}^{\prime} .
\end{gathered}
$$

But the fact that $c=d=0$ implies $Q_{2}^{\prime}=0$ and thus the conserved quantities $Q_{i}$ for the flow (3) imply the conservation of the quantities $Q_{i}^{\prime}$ for the Toda flow. Moreover, using the constants $Q_{i}$, the inverse transform of (13),

$$
\begin{aligned}
& z_{1}=-\frac{e}{y_{3}}, \quad z_{2}=-4\left(y_{4}+i y_{5}\right), \quad z_{3}=-4\left(y_{4}-i y_{5}\right), \\
& z_{4}=\frac{y_{7}-i y_{6}}{y_{4}+i y_{5}}, \quad z_{5}=-\frac{y_{7}+i y_{6}}{y_{4}-i y_{5}}, \quad z_{6}=2 i y_{2},
\end{aligned}
$$

maps the flow (3) into the Toda flow.

Therefore the Toda flow is just another instance of the system (3). It enables one to represent the affine invariant surface as an intersection of quadrics, and it provides a representation of the Toda hyperelliptic curve as a curve of rank 4 quadrics in $\mathbb{P}^{4}$. Finally it shows that the Toda and the Goryachev-Chaplygin flows are intimately related.

\section{References}

1. Adler, M., van Moerbeke, P.: The algebraic integrability of geodesic flow on $S O(4)$. Invent. math. 67, 297-326 (1982), with an appendix by D. Mumford

2. Adler, M., van Moerbeke, P.: The intersection of four quadrics in $\mathbb{P}^{6}$ and its moduli. Math. Ann. (to appear in 1987)

3. Adler, M., van Moerbeke, P.: Kowalewski's asymptotic method, Kac-Moody Lie algebras and regularization. Commun. Math. Phys. 83, 83-106 (1982)

4. Adler, M., van Moerbeke, P.: A systematic approach towards solving integrable systems. Perspectives in Mathematics. New York: Academic Press (to appear in 1987)

5. Chaplygin, S.A.: A new case of rotation of a rigid body, supported at one point (Collected works), Vol. I, Moscow: Gostekhizdat 1948, pp. 118-124

6. Golubev, V.V.: Lectures on the integration of equations of motions of a heavy solid about a fixed point. Moscow: Gostekhizdat 1953 (English translation by Israeli Information agency)

7. Goryachev, D.: On the motion of a rigid material body about a fixed point in the case $A=B=4 C$. Mat. Sb. 21, 3 (1900)

8. van Moerbeke, P.: The spectrum of Jacobi matrices. Invent. math. 37, 45-81 (1976)

9. Guillemin, V., Sternberg, S.: Symplectic techniques in physics. Cambridge, MA: Cambridge University Press 1984

Communicated by A. Jaffe

Received November 10, 1986 